\title{
ENQUETE BIOGRAPHIQUE
}

"MIGRANTS, PARCOURS DE VIE, PARCOURS DE SANTE"

\section{GRILLE BIOGRAPHIQUE}

NOM DE L'ENQUETEUR :

$N^{\circ}$ DE L'ENQUETEUR :

$N^{\circ}$ D'INTER CAPI :

DATE DE L'ENTRETIEN :

$N^{\circ}$ D'ANONYMAT :
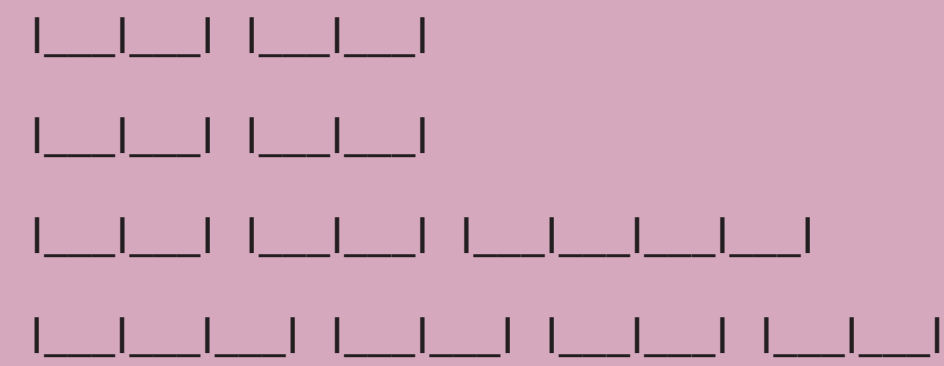


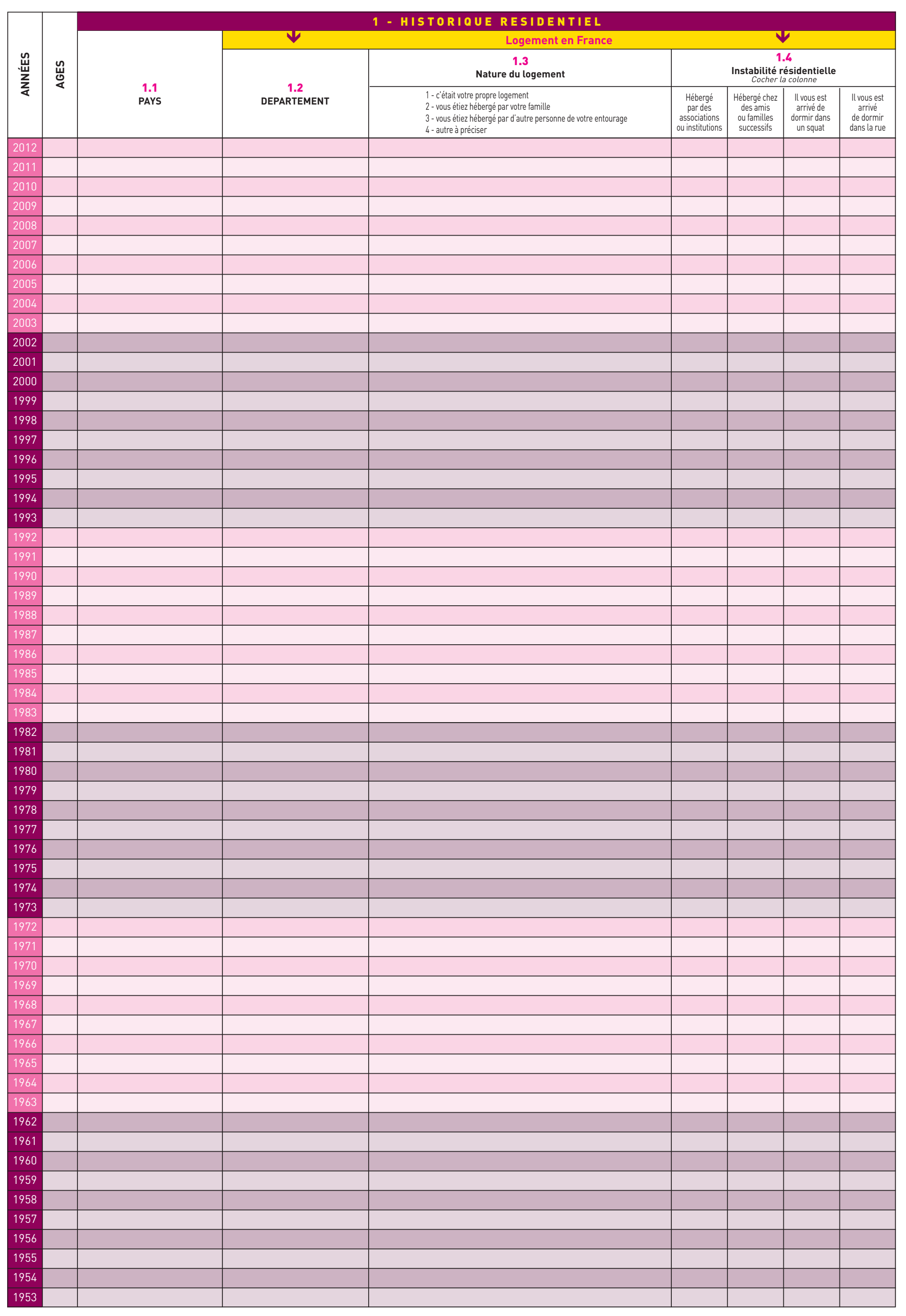




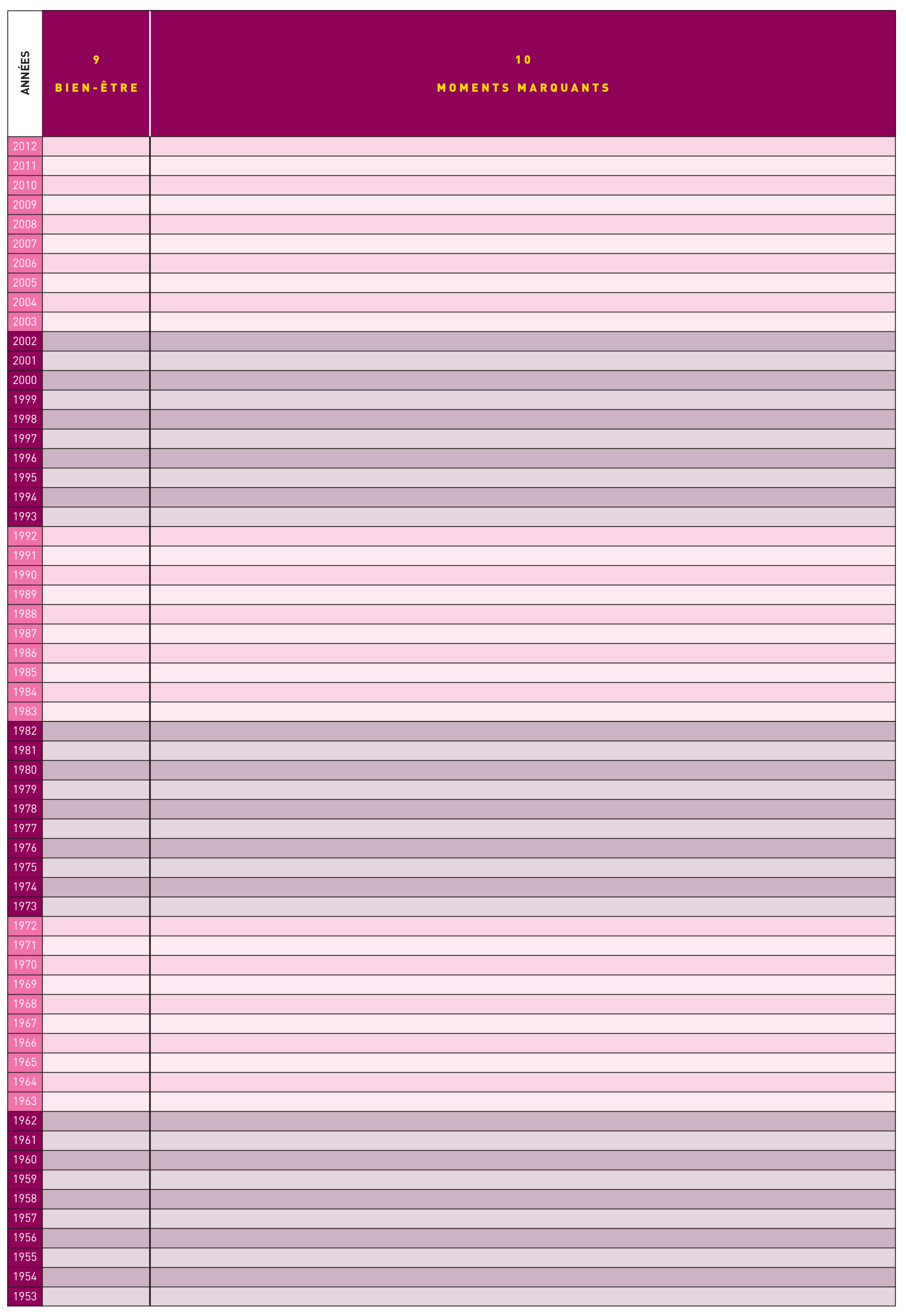


3 - HISTOIRE DES RELATIONS

\begin{tabular}{|c|c|c|}
\hline \multirow{3}{*}{$\begin{array}{l}3.1 \\
\text { RELATIONS LONGUES } \\
\text { (PLUS D'1 AN) }\end{array}$} & \multirow{3}{*}{$\begin{array}{c}3.2 \\
\text { RELATIONS COURTES } \\
\text { OU } \\
\text { OCCASIONNELLES }\end{array}$} & \\
\hline & & \\
\hline & & $\begin{array}{ll}\text { 1- Transactionnelle (TRANSAC) } & \text { Oui / Non / NR } \\
\text { 2- Payés (IAYYSS) } & \text { Oui / Non / NR } \\
\text { 3- Recours prostitution (PAYANTS) Oui / Non / NR } \\
\text { 4- Rapport forcé (FORCES) } & \text { Oui / Non / NR }\end{array}$ \\
\hline
\end{tabular}

2012

2011

2010

2009

2008

2007

2006

2005

2004

2003

2002

2001

1999

1998

1997

1996

1995

1994

1993

1992
4- GROSSESSES et ENFANTS

\section{\begin{tabular}{l|l|l}
4.2 & & \\
4.3 & Ouvitcetenfant
\end{tabular}} \begin{tabular}{l|l|l}
4.3 & Où vit cet enfant actuellement ?
\end{tabular} 4.1 Souhait d'enfant \& grossesse $\quad \begin{gathered}\text { Au moment } \\ \text { où la }\end{gathered}$ - En France avec moi \begin{tabular}{l|l|l|l} 
Enfants & 1 - Vous ne vous posiez pas la question & ous & - Pas du tout \\
& 3 - Voussesse & 2 - En France mais pas avec mo
\end{tabular}

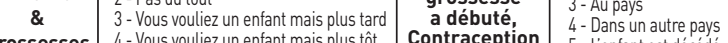

Grossesses 4- Vous vouliez un enfant mais plus tôt Contraception 5 - L'enfant est décédé \begin{tabular}{l|l|l}
\hline - Oui, à ce moment là & $1.0 u i$ & 6 - Autre \\
8 - Non réponse & 2 - Non répons \\
\hline
\end{tabular} \begin{tabular}{|l|l|l}
98 - Non reponse & 2. Non & $\begin{array}{l}98 \text { - Non réponse } \\
99 \text { - Ne sait pas }\end{array}$ \\
\hline
\end{tabular} 


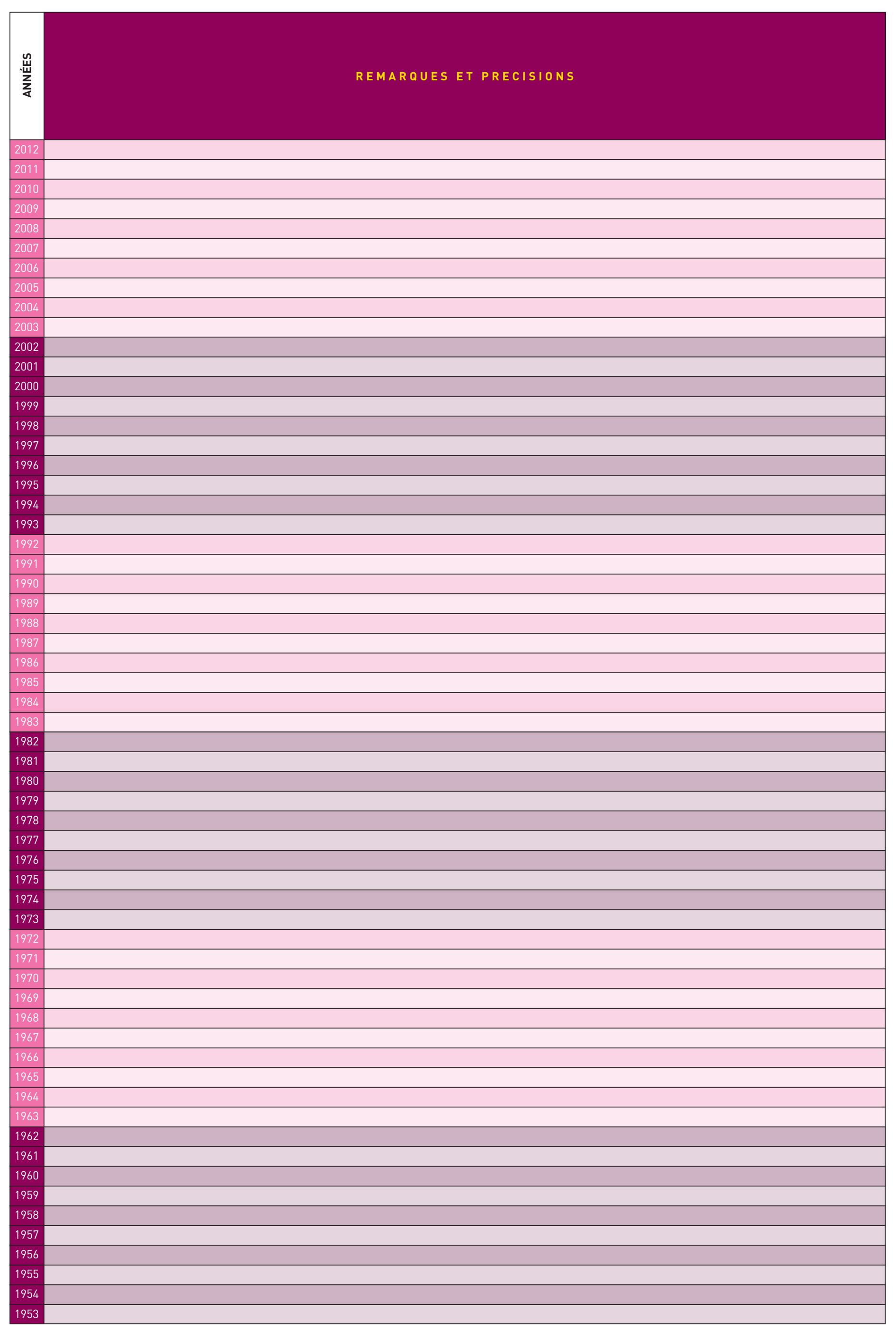




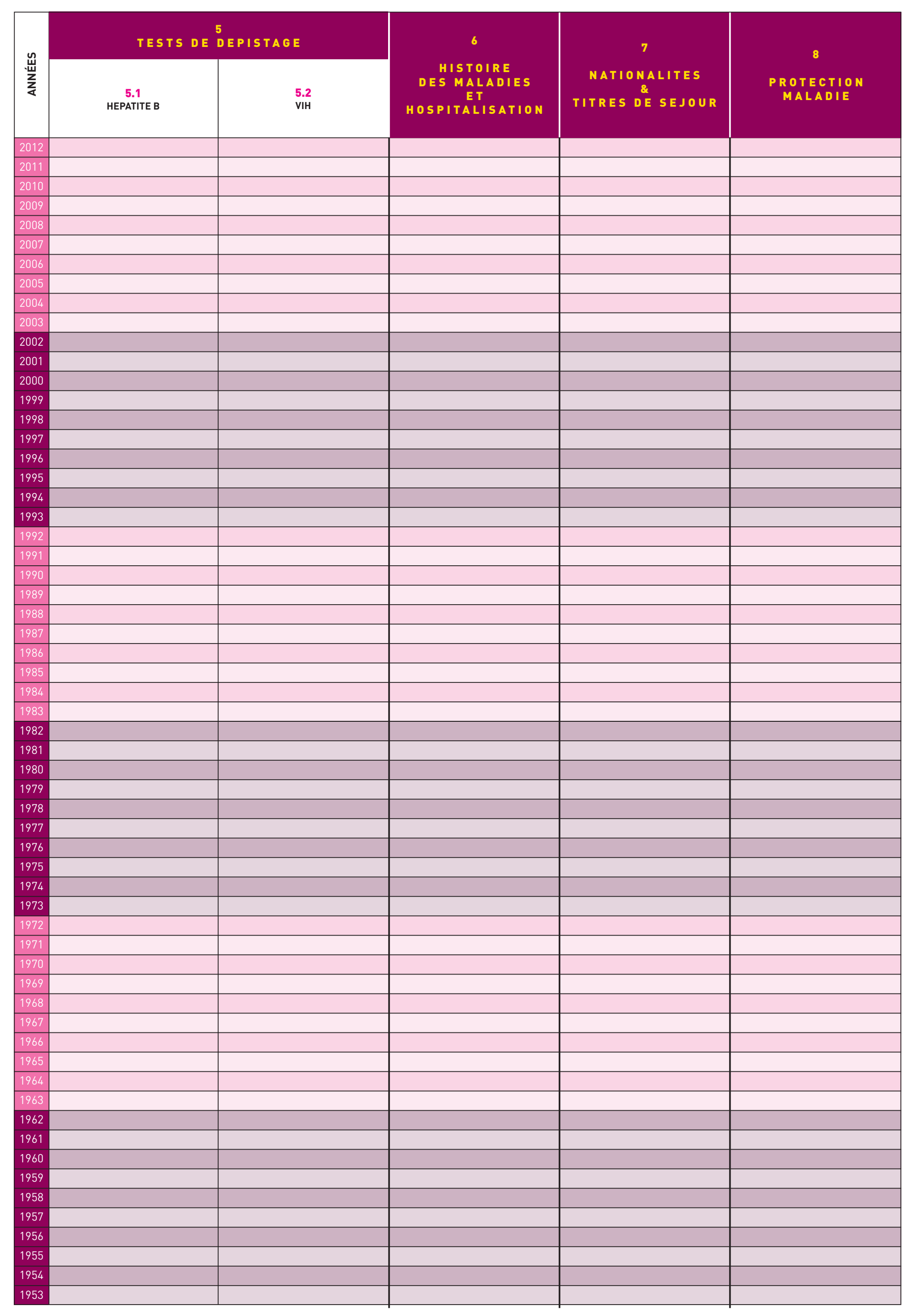


2. HISTORIQUE DES ACTIVITES

2.1

Que faisiez-vous...?

2012

2011

2010

2009

2008

2007

2006

2005

2004

2003

2002

2001

1999

1998

1997

1996

1994

1993

1992

1991

1990

1989

1988

1987

1986

1985

1984

1983

1982

1981

1980

1979

1978

1977

1976

1975

1974

1973

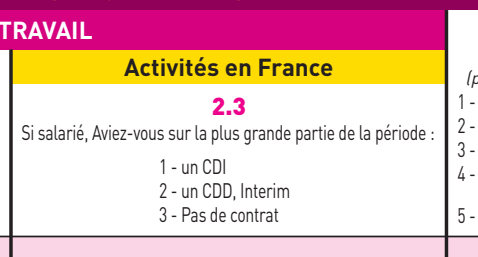

D'où venaient vos ressources? (olusileurs codes possibles pour une même période) - de votre propre activité
2.2

Etiez-vous :

- Cadre $\quad 4$-A son compte Isans salariés -Employe ou ouvrier 5 -Apprenti, stagiaire

3- Patron, employeur 6 -Aide familial.
- d'activités d'autres membres de la famille

- d'allocations de létat l allocation familiale, allocation

de handicas ou invalidite... : A préciser
der 5 - aucune ressource 